\title{
LOCALIZATION OF QUANTUM WAVE PACKETS
}

\author{
ERIC STRÄNG
}

\begin{abstract}
We study the semiclassical propagation of squeezed Gaußian states. We do so by considering the propagation theorem introduced by Combescure and Robert 1 approximating the evolution generated by the Weylquantization of symbols $H$. We examine the particular case when the Hessian $H^{\prime \prime}\left(X_{t}\right)$ evaluated at the corresponding solution $X_{t}$ of Hamilton's equations of motion is periodic in time. Under this assumption, we show that the width of the wave packet can remain small up to the Ehrenfest time. We also determine conditions for "classical revivals" in that case. More generally, we may define recurrences of the initial width. Some of these results include the case of unbounded classical motion. In the classically unstable case we recover an exponential spreading of the wave packet as in [1].
\end{abstract}

\section{INTRODUCTION}

Localization of quantum states in phase space is a prerequisite in some semiclassical treatments of quantum evolution. In the classically chaotic case, the width of an initially localized Gaußian increases exponentially [1] up to the so called Ehrenfest time $T_{E}$, i.e., the time up to which quantum dynamics can be approximated by classical dynamics. In the case of regular classical motion it can be shown that this width grows algebraically in the semiclassical parameter $\hbar$ up to $T_{E}$.

In certain applications, it is necessary to propagate states semiclassically for long times. This demands control of the width of the state. An example is the construction of quasi-modes proposed by Paul and Uribe [2]. One refers to a quasimode as a state $\psi$ which is a solution of a corresponding spectral problem of an operator $\widehat{H}$ up to some small discrepancy $\delta$, i.e.,

$$
\|\widehat{H} \psi-E \psi\|<\delta
$$

which insures, in the case of discrete spectrum, that there exist at least one eigenvalue of $\widehat{H}$ in the interval $[E-\delta, E+\delta]$. The approximation proposed by Paul and Uribe uses the semiclassical propagation of coherent states over closed classical trajectories leading to the well known Bohr-Sommerfeld quantization rule in one dimension. The eigenvalues $E_{n}$ of the Hamiltonian $\widehat{H}$ are given by the quantization condition

$$
\int_{\mathscr{C}_{E_{n}}} p \mathrm{~d} q=2 \pi \hbar\left(n+\frac{1}{2}\right)+\mathcal{O}\left(\hbar^{2}\right), \quad n \in \mathbb{N},
$$

where the energy shells $\mathscr{C}_{E}=\{(p, q): H(p, q)=E\}$ are closed curves.

Certain examples suggest that there exist systems (other than the harmonic oscillator) where the propagated width of an initial Gaußian remains small for long times. Such a behavior is exhibited by the propagation of Gaußians generated by some perturbed periodic Schrödinger operators like the Wannier-Stark Hamiltonian

$$
\widehat{H}_{\mathrm{WS}}(\varepsilon)=-\frac{\hbar^{2}}{2} \Delta_{x}+V_{\Gamma}(x)+\varepsilon x
$$

in the limit of small perturbations $\varepsilon$. Here $V_{\Gamma}(x)$ denotes a periodic potential with respect to a lattice $\Gamma \cong \mathbb{Z}^{d}$. It is known that the band structure of the spectrum of 
the unperturbed operator is preserved for small enough perturbations $\varepsilon$. Numerical studies [3, 4] show that an initially localized Gaußian in momentum space defined on such an energy band apparently remains Gaußian for long times. The evolving states carry out oscillations. The center of the Gaußian can oscillate in position space describing so called Bloch oscillations [5]. Alternatively, the width of the Gaussian can oscillate, whereupon the center remains fixed, describing so called breathing modes. In both cases, the state returns to the initial state after an oscillation period up to a small error.

We study the evolution generated by a class of operators on $L^{2}\left(\mathbb{R}^{d}\right)$ defined as Weyl quantizations of classical symbols $H(X)$ with the property

$$
H^{\prime \prime}\left(X_{t}\right)=H^{\prime \prime}\left(X_{t+T}\right), \quad \forall t \in \mathbb{R},
$$

where $X_{t}$ denotes the solutions to Hamilton's equations of motion and $H^{\prime \prime}(X)$ is the Hessian of $H$ with respect to $X$. In one dimension, the condition (2) is satisfied by bounded classical motion or unbounded motion in a periodic potential. We particularly study the evolution of initial Gaußian (or squeezed) states semiclassically, i.e., asymptotically as $\hbar \searrow 0$, when $t \nearrow \infty$. We focus our attention to the spreading of such wave packets.

In sections 1.1 and 1.2 we shortly review the semiclassical propagation of Gaußian coherent states. In section 1.3 we give some known results on the validity of the approximation. We then make, in section 2.1 and section 2.2 statements about the approximate Gaußian state given by this semiclassical propagation using Floquet theory. These properties are then brought back to the true evolution in section 3 .

1.1. Preliminaries: semiclassical propagation of wave packets. We will work in the context of self adjoint operators defined on $L^{2}\left(\mathbb{R}^{d}\right)$ that are $\hbar$-Weyl quantizations of symbols. To a smooth $\left(C^{\infty}\right)$ classical symbol $b(X)$, i.e., a function on the phase space $T^{*} \mathbb{R}^{d} \cong \mathbb{R}^{2 d}$, there corresponds an operator on $L^{2}\left(\mathbb{R}^{d}\right)$, $\widehat{b}:=\mathrm{Op} \mathrm{p}_{\hbar}^{w}[b]$, defined by

$$
\mathrm{Op}_{\hbar}^{w}[b] \psi(x):=\frac{1}{(2 \pi \hbar)^{d}} \int_{\mathbb{R}^{2 d}} b\left(\frac{x+y}{2}, \xi\right) \psi(y) e^{\frac{\mathrm{i}}{\hbar}(x-y) \xi} \mathrm{d} y \mathrm{~d} \xi .
$$

The following conditions will be assumed :

(1) The classical Hamiltonian $H: \mathbb{R}^{2 d} \rightarrow \mathbb{R}$ is a smooth function.

(2) $H \in S(m)$, i.e., for all multi-indices $\alpha, \beta$, there exists $K_{\alpha, \beta}>0$ such that

$$
\left|\partial_{p}^{\alpha} \partial_{q}^{\beta} H(p, q)\right| \leq K_{\alpha, \beta}\left(1+|p|^{2}+|q|^{2}\right)^{\frac{m}{2}} .
$$

(3) The corresponding classical equation of motion is given by

$$
\frac{\mathrm{d} X_{t}}{\mathrm{~d} t}=\mathcal{J} H^{\prime}\left(X_{t}\right)
$$

where $\mathcal{J}$ is the symplectic unity

$$
\mathcal{J}:=\left(\begin{array}{cc}
0 & -\mathbb{1}_{d \times d} \\
\mathbb{1}_{d \times d} & 0
\end{array}\right)
$$

and $H^{\prime}$ is the gradient of $H$ with respect to $X$. Furthermore, we denote by $\Phi_{H}^{t}: \mathbb{R}^{2 d} \rightarrow \mathbb{R}^{2 d}, X_{0} \mapsto X_{t}=\Phi_{H}^{t}\left(X_{0}\right)$ the corresponding classical flow.

(4) The $\hbar$-Weyl quantization of $H(X), \widehat{H}:=\mathrm{Op}_{\hbar}^{w}[H]$, is an essentially selfadjoint operator on $L^{2}\left(\mathbb{R}^{d}\right)$ and generates a unitary time evolution $\forall t \in \mathbb{R}$,

$$
\begin{aligned}
\widehat{U}(t) \quad: \quad & L^{2}\left(\mathbb{R}^{d}\right) \rightarrow L^{2}\left(\mathbb{R}^{d}\right) \\
& \psi(0) \mapsto \psi(t)
\end{aligned}
$$


corresponding to the Schrödinger equation

$$
\mathrm{i} \hbar \frac{\partial \psi}{\partial t}=\widehat{H} \psi
$$

i.e., we will write $\widehat{U}(t)=e^{-\frac{i}{\hbar} \widehat{H} t}$.

The Weyl calculus also allows a representation of quantum mechanical wave functions on phase space. This is given by the Wigner function of the state $u \in$ $L^{2}\left(\mathbb{R}^{d}\right)$,

$$
W[u](p, q):=\int_{\mathbb{R}^{d}} \bar{u}\left(q+\frac{y}{2}\right) u\left(q-\frac{y}{2}\right) e^{\frac{i}{\hbar} p y} \mathrm{~d} y .
$$

The Wigner function of a Gaußian defines a positive measure on phase space.

1.2. A semiclassical propagation theorem. With these assumptions we give a short summary of the method of semiclassical propagation of coherent states introduced by Combescure and Robert [1. Similar constructions have also been considered in the past. See, e.g., Hagedorn [6, 7] and references therein.

The idea is to expand the exact Hamiltonian $\widehat{H}$ along the classical flow generated by the symbol $H$ up to second order, whereupon the approximate time dependent Hamiltonian, $\widehat{H}_{2}(t)$, is the $\hbar-$ Weyl quantization of

$$
H_{2}(t, Y):=H\left(X_{t}\right)+\left(Y-X_{t}\right)^{T} H^{\prime}\left(X_{t}\right)+\frac{1}{2}\left(Y-X_{t}\right)^{T} H^{\prime \prime}\left(X_{t}\right)\left(Y-X_{t}\right) .
$$

The propagation of normalized wave functions (squeezed states) $)^{1}$ of the form

$$
(5) \psi_{Z}^{(p, q)}(x):=\frac{\operatorname{det}(\Im(Z))^{\frac{1}{4}}}{(\pi \hbar)^{\frac{d}{4}}} e^{\frac{i}{\hbar}\left(p^{T}(x-q)+(x-q)^{T} \frac{Z}{2}(x-q)\right)}, Z \in \Sigma_{d},\left(\begin{array}{c}
p \\
q
\end{array}\right) \in \mathbb{R}^{2 d}
$$

by quadratic Hamiltonians is well known [8]. By $\Sigma_{d}$, we mean the $d$-dimensional Siegel upper half space, i.e., the set of symmetric $d \times d$ matrices with positive, nondegenerate imaginary part [8]. The quadratic form $Z$ describes the shape of the wave packet and it should be underlined that it is independent of $\hbar$. In its Wigner representation (see eq.(4) $) \psi_{Z}^{(p, q)}$ is a Gaußian centered around $X=\left(\begin{array}{c}p \\ q\end{array}\right)$. This Wigner function is given by

$$
W\left[\psi_{Z}^{X}\right](Y)=\left(\frac{1}{\pi \hbar}\right)^{d} \exp \left(-\frac{1}{\hbar}(Y-X)^{T} G(Y-X)\right)
$$

where

$$
G:=\left(\begin{array}{cc}
\Im(Z)^{-1} & -\Im(Z)^{-1} \Re(Z) \\
-\Re(Z) \Im(Z)^{-1} & \Im(Z)+\Re(Z) \Im(Z)^{-1} \Re(Z)
\end{array}\right)
$$

is independent of $\hbar$.

The unitary evolution, $\widehat{U}_{2}(t)$, generated by $\widehat{H}_{2}(t)$, acts on a squeezed state by translation and metaplectic action, i.e.,

$$
\widehat{U}_{2}(t)=e^{\frac{i}{\hbar} \Theta(t)} \widehat{\mathcal{T}}\left(X_{t}\right) \widehat{\mathcal{M}}\left(S_{t}\right)
$$

\footnotetext{
${ }^{1}$ We will, with some lack of rigor, call these states Gaußian, coherent or squeezed without distinction.
} 
where $\widehat{\mathcal{T}}$ is the translation operator on $\mathbb{R}^{2 d}$, i.e., $\forall Y \in \mathbb{R}^{2 d},=:\left(\begin{array}{c}\xi \\ q\end{array}\right)$ and $u \in$ $L^{2}\left(\mathbb{R}^{d}\right)$ we have

$$
\begin{aligned}
\widehat{\mathcal{T}}(Y) W[u](Z) & :=e^{\frac{\mathrm{i}}{\hbar}\left(\xi^{T} \widehat{x}-q^{T} \widehat{p}\right)} W[u](Z) \\
& =W[u](Z-Y)
\end{aligned}
$$

where we denote by $\widehat{p}$ the momentum operator and by $\widehat{x}$ the position operator. The metaplectic operator $\widehat{\mathcal{M}}(F)$ is the quantization of a linear symplectomorphism on $\mathbb{R}^{2 d}$ given by the symplectic matrix $F$. These operators form a double-valued unitary representation 2 of the linear symplectomorphism of $\mathbb{R}^{2 d}$. $S_{t}$ denotes the flow differential. The classical flow $\Phi_{H}^{t}$ is a symplectomorphism which ensures that the flow differential is a symplectic matrix. Furthermore, $S_{t}$ satisfies

$$
\begin{aligned}
\frac{\mathrm{d} S_{t}}{\mathrm{~d} t} & =\mathcal{J} H^{\prime \prime}\left(X_{t}\right) S_{t}, \\
S_{0} & =\mathbb{1}_{2 d \times 2 d} .
\end{aligned}
$$

In the prefactor of eq. (7) we have used

$$
\Theta(t):=\mathcal{W}(t)+\hbar \mu,
$$

where

$$
\mathcal{W}(t):=\int_{0}^{t}\left(p_{\tau}^{T} \dot{q}_{\tau}-H\left(p_{\tau}, q_{\tau}\right)\right) d \tau,
$$

is the action of the classical trajectory and $\mu$ is the Maslov index of the classical trajectory. We have here expressed the solution of Hamilton's equations in terms of the canonical variables

$$
\left(\begin{array}{c}
p_{t} \\
q_{t}
\end{array}\right):=X_{t}
$$

Acting with (7) on (5), one obtains at time $t$ a new Gaußian state $e^{\frac{i}{\hbar} \Theta(t)} \psi_{Z_{t}}^{X_{t}}$ up to a phase. The Gaußian is centered around $X_{t}$ in phase space and has a quadratic form $Z_{t} \in \Sigma_{d}$, is given explicitly by the group action [8]

$$
\begin{aligned}
Z_{t} & =S_{t}\left[Z_{0}\right] \\
& =\left(A_{t} Z_{0}+B_{t}\right)\left(C_{t} Z_{0}+D_{t}\right)^{-1}, Z_{0} \in \Sigma_{d},
\end{aligned}
$$

i.e., linear fractional transformation on $\Sigma_{d}$. The matrix $S_{t}$ is here written by means of the $d \times d$ blocks $A_{t}, B_{t}, C_{t}$ and $D_{t}$, i.e.,

$$
S_{t}=\left(\begin{array}{cc}
A_{t} & B_{t} \\
C_{t} & D_{t}
\end{array}\right)
$$

The quadratic form of the Wigner transform of $\psi_{Z_{t}}^{X_{t}}$, is explicitly given by

$$
G_{t}=\left(S_{t}^{-1}\right)^{T} G_{0} S_{t}^{-1} \text {. }
$$

The evolution of the approximate wave packet can be perceived as being generated by rotation and scaling of the Gaußian profile in $\mathbb{R}^{2 d}$.

The difficulty of this scheme resides in the control of errors made by the approximation of $\widehat{U}(t)$ in terms of $\widehat{U}_{2}(t)$. We do not dwell on the details, but just state the result (for proof and a thorough description see [1]). The approximation can be

\footnotetext{
${ }^{2} \mathrm{~A}$ thorough description of the action of the evolution generated by quadratic operators on Gaußians and the metaplectic representation can be found in 8 .
} 
checked to all orders in $\hbar$. For this purpose, one defines the following approximating state

$$
\Psi^{(N)}(t, x):=e^{\frac{i}{\hbar} \Theta(t)} \sum_{0 \leq j \leq N} \hbar^{\frac{j}{2}} \pi_{j}\left(X_{t}, \frac{x}{\sqrt{\hbar}}, t\right) \psi_{Z_{t}}^{X_{t}}(x),
$$

where $\pi_{j}\left(X_{t}, \frac{x}{\sqrt{\hbar}}, t\right)$ are polynomials in $x / \sqrt{\hbar}$ and $X_{t}$ of degree smaller than or equal to $3 j$ with time dependent coefficients.

Theorem 1. (Combescure, Robert) Under the above mentioned assumptions and for an initial Gaußian state $\psi_{Z_{0}}^{X_{0}}$ centered in the phase space representation at $X_{0} \in$ $\mathbb{R}^{2 d}$, for every $N \in \mathbb{N}$ there exists $C<\infty$ such that $\forall \hbar \in\left(0, \hbar_{0}\right], \hbar_{0}>0$,

$$
\left\|\widehat{U}(t) \psi_{Z_{0}}^{X_{0}}-\Psi^{(N)}(t)\right\| \leq C_{N} \hbar^{\frac{N+1}{2}} t e^{3 \gamma t}
$$

where $0 \leq \gamma<\infty$ is the Lyapunov exponent of the classical motion.

We recall that a Lyapunov exponent is a measure of the exponential stability of the solutions of a differential equation upon change of initial conditions. In the case of classical motion, this is given by the Lyapunov exponent defined as

$$
\gamma:=\max _{k}\left[\lim _{t \rightarrow \infty} \sup \left(\frac{\ln \left(s_{k}(t)\right)}{t}\right)\right]
$$

where $s_{k}(t)$ are the singular values of $S_{t}$. The Lyapunov exponent $\gamma$ hence satisfies

$$
\left\|S_{t}\right\|_{\mathrm{HS}}<c_{0} e^{\gamma|t|}
$$

where $c_{0}<\infty$ is a positive constant. We denote by $\|M\|_{\mathrm{HS}}=\sqrt{\operatorname{tr}\left(M^{\dagger} M\right)}$ the Hilbert-Schmidt norm of the matrix $M$. Hermitian conjugation is denoted by $M^{\dagger}$, and transposition by $M^{T}$.

1.3. Ehrenfest time and spreading of wave packets. It is customary, in this context, to define what is known as the Ehrenfest time. The latter is a time scale up to which the above approximation is valid. We define the Ehrenfest time, denoted $T_{E}(\hbar)$, as the maximal time up to which

- the error $\left\|\widehat{U}(t) \psi_{Z_{0}}^{X_{0}}-e^{\frac{i}{\hbar} \Theta(t)} \psi_{Z_{t}}^{X_{t}}\right\|$ remains small,

- the exact state remains localized.

The latter ensures that the approximation retains a physical meaning, i.e., the above classical approximation makes no sense if the state does not remain localized.

The total width of the semiclassically evolved Gaußian is

$$
\sigma_{t}\left(X_{0} ; \hbar\right):=\Delta x^{2}(\hbar, t)+\Delta p^{2}(\hbar, t)=\frac{\hbar}{2} \operatorname{tr}\left(G_{t}\right),
$$

where we have used the variance

$$
\Delta x^{2}=\left\langle e^{\frac{i}{\hbar} \Theta(t)} \psi_{Z_{t}}^{X_{t}}, \widehat{x}^{2} e^{\frac{i}{\hbar} \Theta(t)} \psi_{Z_{t}}^{X_{t}}\right\rangle_{L^{2}\left(\mathbb{R}^{d}\right)}-\left\langle e^{\frac{i}{\hbar} \Theta(t)} \psi_{Z_{t}}^{X_{t}}, \widehat{x} e^{\frac{i}{\hbar} \Theta(t)} \psi_{Z_{t}}^{X_{t}}\right\rangle_{L^{2}\left(\mathbb{R}^{d}\right)}^{2},
$$

and similarly for the momentum operator.

From eq. (10) one obtains that

$$
\sigma_{t} \leq \sigma_{0}\left\|S_{t}\right\|_{\mathrm{HS}}^{2} .
$$

By Theorem [1 there will exist a constant $c_{1}$ such that

$$
\left\|\widehat{U}(t) \psi_{Z_{0}}^{X_{0}}-e^{\frac{i}{\hbar} \Theta(t)} \psi_{Z_{t}}^{X_{t}}\right\| \leq c_{1} \sqrt{\hbar} t e^{3 \gamma t} .
$$

The time scale for which the errors are small is thus algebraic in $\hbar$ if $\gamma=0$. In the generic case, the errors remain small for logarithmic times in $\hbar$. 
With this result, the errors

$$
(15) \Delta(t):=\left\langle\widehat{U}(t) \varphi_{0}, \widehat{s}\left(\widehat{U}(t) \varphi_{0}\right)\right\rangle_{L^{2}\left(\mathbb{R}^{d}\right)}-\left\langle\widehat{U}_{2}(t) \varphi_{0}, \widehat{s}\left(\widehat{U}_{2}(t) \varphi_{0}\right)\right\rangle_{L^{2}\left(\mathbb{R}^{d}\right)}
$$

for propagating observables $\widehat{s}$ can be approximated explicitly. Of particular interest to us is the width operator $\widehat{s}:=\mathrm{Op}_{\hbar}^{w}\left[|Y|^{2}\right]$. One can characterize the times for which the error $\Delta(t)$ is small, e.g., $\Delta(t)=\mathcal{O}\left(\hbar^{\alpha}\right)$ for some $\alpha>0$. Again, using the Lyapunov inequality (13) one finds that the width of the approximate state is bounded by

$$
\sigma_{t}(\hbar) \leq c_{3} e^{2 \gamma|t|}, \quad c_{3}>0,
$$

and the error remains $\mathcal{O}\left(\hbar^{\alpha}\right)$ up to times of order $\frac{|\ln (\hbar)|}{6 \gamma}$. We may thus generically state that the Ehrenfest time is

$$
T_{E}(\hbar) \propto \frac{|\ln (\hbar)|}{6 \gamma} .
$$

In the integrable case, implying $\gamma=0$, the width grows like the square of the Hilbert-Schmidt norm of the flow differential, i.e., at most polynomially in time. The error remains small for times up to $\hbar^{-\frac{1}{2}}$. The Ehrenfest time thus is algebraic in $\hbar$.

Our aim is to characterize the spreading of the approximate state for a more specific class of classical motions.

\section{Results}

In addition to the conditions imposed on symbols $H$ above (see section 1.1), we assume that the Hessian $H^{\prime \prime}\left(X_{t}\right)$ is $T$-periodic, i.e.,

$$
H^{\prime \prime}\left(X_{t}\right)=H^{\prime \prime}\left(X_{t+T}\right), \quad \forall t \in \mathbb{R} .
$$

We will also utilize the following definition.

Definition 1. A classical revival at a time $t>0$ is the event that the approximate Gaußian given above is the initial one up to a phase factor, i.e.,

$$
\psi_{Z_{t}}^{X_{t}}=e^{\mathrm{i} \alpha_{t}} \psi_{Z_{0}}^{X_{0}}
$$

2.1. Floquet theory. According to the Floquet theorem [9, any linear differential equation with continuous $T$-periodic coefficients has a periodic solution of the second type, i.e., a solutions which satisfy

$$
f(t+T)=v f(t), \quad v \in \mathbb{C}, \forall t \in \mathbb{R} .
$$

In particular, the linear vector differential equation

$$
\frac{\mathrm{d}}{\mathrm{d} t} f=A(t) f
$$

where $A(t)$ is continuous and satisfies $A(t+T)=A(t), \forall t \in \mathbb{R}$, has a fundamental matrix of the form (see e.g. [10])

$$
F_{t}=M^{-1} e^{L t} M U(t), \quad M \in \operatorname{GL}(n, \mathbb{C}),
$$

where $L$ is a diagonal matrix and $U(t)$ is a $T$-periodic matrix. By definition, a fundamental matrix is a full rank matrix whose columns satisfy the differential equation, i.e., the linear combinations of the columns of the fundamental matrix span the full space of solutions of the differential equation.

We will call the elements of $L$ the Floquet exponents of the fundamental system.

The result of this section will be summarized in the following way. 
Proposition 1. If $H^{\prime \prime}\left(X_{t}\right)$ is periodic and the Floquet exponents of $S_{t}$ are purely complex then the width of a Gaußian propagated semiclassically by $\widehat{U}_{2}(t)$ will remain unchanged at multiples of the smallest classical period T. Furthermore, if the classical flow is periodic then classical revivals will occur.

Proof . Under the condition (17), Floquet theory states the existence of a fundamental Floquet matrix for the linear differential eq. (8),

$$
S_{t}=M^{-1} e^{\Lambda t} M F_{t}, \quad M \in \mathrm{GL}(2 d, \mathbb{C}),
$$

where $e^{\Lambda t}$ is the diagonal matrix with entries $e^{2 \pi \lambda_{i} \frac{t}{T}}, \lambda_{i} \in \mathbb{C}, F_{t}$ has minimal period $T$, and we have chosen

$$
F_{0}=S_{0}=\mathbb{1}_{2 d \times 2 d}
$$

One directly concludes that

$$
S_{k T}=M^{-1} e^{k T \Lambda} M, \quad \forall k \in \mathbb{Z} .
$$

for multiples $k$ of the classical period $T$.

Furthermore, the real fundamental matrix defined by $S_{t} \in \mathrm{Sp}(2 d, \mathbb{R})$ has a unique polar decomposition, i.e., there exists [8] an orthogonal matrix $\mathcal{Q}_{t} \in \mathrm{O}(2 d) \cap$ $\operatorname{Sp}(2 d, \mathbb{R})$ and a positive definite matrix $\mathcal{P}_{t} \in \operatorname{Sp}(2 d, \mathbb{R})$ such that

$$
S_{t}=\mathcal{Q}_{t} \mathcal{P}_{t} .
$$

The width of the approximate squeezed state at time $t$ is (see eq.

$$
\sigma_{t}(\hbar)=\operatorname{tr}\left(\left(S_{t}^{-1}\right)^{T} G_{0} S_{t}^{-1}\right),
$$

and since any symplectic matrix $A$ satisfies 8

$$
A^{-1}=\mathcal{J} A^{T} \mathcal{J}^{-1},
$$

we can write by eq. (10),

$$
\sigma_{t}(\hbar)=\frac{\hbar}{2} \operatorname{tr}\left(\mathcal{Q}_{t} \mathcal{P}_{t} \mathcal{J}^{T} G_{0} \mathcal{J} \mathcal{P}_{t}^{T} \mathcal{Q}_{t}^{T}\right)=\frac{\hbar}{2} \operatorname{tr}\left(\mathcal{P}_{t} \mathcal{J}^{T} G_{0} \mathcal{J} \mathcal{P}_{t}^{T}\right),
$$

since $\mathcal{Q}_{t} \in \mathrm{O}(2 d)$.

We are hence confronted with two cases.

- Either $S_{k T}$ is orthogonal, i.e., $\mathcal{P}_{k T}=\mathbb{1}_{2 d \times 2 d}$; this corresponds to

$$
\sigma_{k T}(\hbar)=\frac{\hbar}{2} \operatorname{tr}\left(G_{0}\right)=\sigma_{0}(\hbar), \quad \forall k \in \mathbb{Z} .
$$

The orthogonality of $S_{k T}$ implies that it has $2 d$ singular values 1 . It is furthermore similar to $e^{k T \Lambda}\left(F_{k T}=\mathbb{1}_{2 d \times 2 d}\right)$, which hence is unitary so the Floquet exponents $\frac{2 \pi \lambda_{i}}{T}$ are purely complex or zero. By definition, the Lyapunov exponent of the classical trajectories is

$$
\gamma:=\max _{k}\left[\lim _{t \rightarrow \infty} \sup \left(\frac{\ln \left(s_{k}\left(X_{t}\right)\right)}{t}\right)\right],
$$

where $s_{k}(t)$ are the singular values of the flow differential $S_{t}$, i.e., the eigenvalues of

$$
F_{t}^{\dagger}\left(M^{-1} e^{t \Lambda} M\right)^{\dagger} M^{-1} e^{t \Lambda} M F_{t}=F_{t}^{\dagger} F_{t} .
$$

Noting that $F_{t}$ is bounded (and periodic), we see directly that if

$$
\max _{i}\left(\Re\left(\lambda_{i}\right)\right)=0,
$$

the classical motion is linearly stable. 
- $\mathcal{P}_{k T} \neq \mathbb{1}_{2 d \times 2 d}$, which corresponds to

$$
\begin{aligned}
\sigma_{k T}(\hbar) & =\frac{\hbar}{2} \operatorname{tr}\left(\mathcal{P}_{k T} \mathcal{J}^{T} G_{0} \mathcal{J} \mathcal{P}_{k T}^{T}\right) \\
& >\sigma_{0}(\hbar) .
\end{aligned}
$$

since $\mathcal{P}_{t} \in \mathrm{Sp}(2 d, \mathbb{R})$ is strictly positive definite. This corresponds to the case when $\Re\left(\lambda_{i}\right) \neq 0$, hence $\max _{i}\left(s_{i}\right)>0$. The classical motion is hence not linearly stable in this case.

Furthermore, if the (purely complex) Floquet exponents are rationally dependent, there exist some multiples

$$
T_{R}=n_{R} T, \quad n_{R} \in \mathbb{Z},
$$

of the classical period $T$ such that the orthogonal transformation at times $T_{R}$ reduces to unity. Indeed, if $n_{R}$ is the smallest common multiple of the denominators of the sequence $\left\{\lambda_{i}\right\}$ defined by the Floquet exponents, i.e.,

$$
\lambda_{i} n_{R} \in \mathbb{N}, \forall i,
$$

we find

$$
\begin{aligned}
M^{-1} e^{\Lambda T_{R}} M & =M^{-1} \operatorname{diag}\left\{e^{2 \pi \mathrm{i} n_{i}}\right\} M, \quad n_{i} \in \mathbb{Z}, \\
& =\mathbb{1}_{2 d \times 2 d} .
\end{aligned}
$$

The semiclassical approximation then is the initial Gaußian, if it is localized at the initial point $X_{0}$. This is the case if the flow is periodic. In this case we have a classical revival.

Remark 1. Note that these revivals are purely classical in the sense that the conditions only reflect properties of the classical motion and are hence independent of $\hbar$.

Remark 2. If $S_{k T}$ is orthogonal, the approximate Gaußian profile will be the initial one at classical periods. It is just rotated and translated in phase space.

2.2. A uniform bound. We can further characterize the approximate state in the case when the Floquet exponents are purely complex.

Lemma 1. If $H^{\prime \prime}\left(X_{t}\right)$ is T-periodic and the Floquet exponents of $S_{t}$ are purely complex then the width $\sigma_{t}(\hbar)$ of a Gaußian state propagated by $\widehat{U}_{2}(t)$ satisfies the following uniform bound

$$
\sigma_{t}(\hbar) \leq K \sigma_{0}(\hbar), \forall t \in \mathbb{R} .
$$

Furthermore, $K:=e^{\kappa}$, where $\kappa$ is defined by

$$
\kappa:=2 T \sup _{t \in[0, T]}\left\|\mathcal{J} H^{\prime \prime}\left(X_{t}\right)\right\|_{\mathrm{HS}} .
$$

Proof . We consider eq. (8)

$$
\left\{\begin{aligned}
\frac{\mathrm{d} S_{t}}{\mathrm{~d} t} & =\mathcal{J} H^{\prime \prime}\left(X_{t}\right) S_{t} \\
S_{0} & =\mathbb{1} .
\end{aligned}\right.
$$

Starting at some initial time $k T, k \in \mathbb{Z}, S_{t+k T}$ satisfies the Grönwall inequality

$$
\left\|S_{t+k T}\right\|_{\mathrm{HS}} \leq\left\|S_{k T}\right\|_{\mathrm{HS}} e^{\kappa}, \forall t \in[0, T],
$$

during a classical period $T$ where

$$
\kappa=2 T \sup _{t \in[0, T]}\left\|\mathcal{J} H^{\prime \prime}\left(X_{t}\right)\right\|_{\mathrm{HS}} .
$$


Furthermore,

$$
\begin{aligned}
\sigma_{t}(\hbar) & =\frac{\hbar}{2} \operatorname{tr}\left(S_{t} \mathcal{J}^{T} G_{0} \mathcal{J} S_{t}^{T}\right) \\
& =\frac{\hbar}{2} \operatorname{tr}\left(G_{0} S_{t}^{T} S_{t}\right) \leq \frac{\hbar}{2}\left|\operatorname{tr}\left(G_{0}\right)\right|\left\|S_{t}\right\|_{\mathrm{HS}}^{2}
\end{aligned}
$$

Hence

$$
\sigma_{t}(\hbar) \leq \frac{\hbar}{2}\left|\operatorname{tr}\left(\mathbf{g}_{0}\right)\right| e^{\kappa}
$$

if $S_{t}$ has purely complex Floquet exponents since in this case $\sigma_{0}(\hbar)=\sigma_{k T}(\hbar)$, according to proposition 1 .

We conclude with this choice that

$$
\sigma_{t}(\hbar) \leq K \sigma_{0}(\hbar)
$$

uniformly in time.

If $S_{t}$ is not orthogonal at the classical periods $T$, the latter bound takes the form

$$
\sigma_{t}(\hbar) \leq K \sigma_{0}(\hbar) e^{2 \max _{i}\left(\Re\left(l_{i}\right)\right)|t|}
$$

where $l_{i}:=\frac{2 \pi \lambda_{i}}{T}$ are the corresponding Floquet exponents of $S_{t}$ which is nothing else than a Lyapunov inequality where $\max _{i}\left(\Re\left(l_{i}\right)\right)$ plays the role of the Lyapunov exponent. Using the fact that what we are approximating is the Hilbert-Schmidt norm of the flow differential one can furthermore state the existence of a constant $c_{4}>0$ such that

$$
\frac{e^{2 \max _{i}\left(\Re\left(l_{i}\right)\right)|t|}}{c_{4}} \leq \sigma_{t}(\hbar) \leq c_{4} e^{2 \max _{i}\left(\Re\left(l_{i}\right)\right)|t|} .
$$

which determines the asymptotic behavior of the width in that case.

Remark 3. We note that the equality in eq. (18) is reached if the classical period is null, i.e., if the Hamiltonian has a constant Hessian $H^{\prime \prime}\left(X_{t}\right)$. This is satisfied by the harmonic oscillator. The flow differential $S_{t}$ is then an orthogonal matrix for all times. It is well known that the propagation is dispersion-less in this case.

\section{Discussion}

Theorem 1 allows us to trace back the properties of the approximation to the exact state $\mathcal{U}(t) \psi_{Z_{0}}^{X_{0}}$. According to the discussion in [1] (see also section 1.3), this can be done with an error of order $\mathcal{O}\left(\hbar^{\alpha}\right), \alpha>0$ up to times $\hbar^{-\frac{1}{2}}$ if the Floquet exponents have zero real part (stable classical dynamics) and up to times $\frac{|\ln (\hbar)|}{6 \nu}, \nu=\max _{i} \Re\left(l_{i}\right)$ otherwise (unstable classical dynamics) as $\hbar \searrow 0$ and $t \nearrow \infty$.

In the stable case, the approximate state remains localized ad infinitum if this state remains localized between classical periods since the width of such a state is the same at classical periods. We wish to stress that we have defined the classical period as the minimal period of the Hessian $H^{\prime \prime}\left(X_{t}\right)$ which is not necessarily the period of the classical flow $\Phi_{H}^{t}$. An example is classical motion in a one dimensional periodic potential $V_{\Gamma}$ with energies $E$ such that

$$
E>\sup _{x \in \mathbb{R}}\left(V_{\Gamma}(x)\right) \text {. }
$$

In such a case $H^{\prime \prime}\left(X_{t}\right)$ is periodic although the flow $\Phi_{H}^{t}\left(X_{0}\right)$ is not. Furthermore, the approximation remains localized between classical periods if

$$
\kappa=2 T \sup _{t \in[0, T]}\left\|\mathcal{J} H^{\prime \prime}\left(X_{t}\right)\right\|_{\mathrm{HS}},
$$


the exponent in eq. (19), is small enough. We can hence state in that case, up to small errors $\mathcal{O}\left(\hbar^{\alpha}\right)$, localization of the exact propagation up to the Ehrenfest time $T_{E} \propto \hbar^{-\frac{1}{2}}$. Note that the situation includes unbounded motion.

If the flow differential $S_{t}$ is periodic, it is clear that the shape will be the initial one at classical periods up to a small error. The resonance condition allows to state that this will also occur at some time if the flow differential is merely periodic of the second type and if the Floquet exponents are rationally dependent and purely imaginary. We may state this since the exact state remains localized and that our asymptotic considerations are valid as $\hbar \searrow 0$ and $t \nearrow \infty$. The property extends also to the general stable case, i.e., in the case of rationally independent Floquet exponents. Recall that the quadratic form $Z_{t}$ is given by linear fractional transformation. In particular,

$$
Z_{k T}=\left(A_{k T} Z_{0}+B_{k T}\right)\left(C_{k T} Z_{0}+D_{k T}\right)^{-1}
$$

where we have used the notation of section 1.2. Since $S_{k T} \sim e^{\Lambda k T}$ which is unitary with eigenvalues $e^{i \ell_{i} \frac{2 \pi}{T}}=e^{\lambda_{i} \frac{2 \pi}{T}}$ where $\ell_{i} \in \mathbb{R}$, there exist $k$-independent matrices $a_{i} \in \mathbb{C}^{d \times d}$ such that $Z_{k T}$ can be viewed as the image of the vector $\ell \in \mathbb{T}^{d}$ with entries $\ell_{i}$ under the continuous map

$$
\begin{aligned}
\mathrm{e}: \mathrm{T}^{d} & \rightarrow \Sigma_{d} \\
\omega & \mapsto \sum_{i=0}^{d} a_{i} e^{2 \pi \mathrm{i} \omega_{i}} .
\end{aligned}
$$

Furthermore, the endomorphism

$$
\begin{aligned}
\tau: & \mathbb{T}^{d} \rightarrow \mathbb{T}^{d} \\
& L \mapsto L+\ell,
\end{aligned}
$$

where $\left(L_{i}+\ell_{i}\right)$ is defined modulo 1 , is known to be ergodic with respect to Lebesgue measure on the $d$-torus since the frequencies $\ell_{i}$ are rationally independent. In this notation we have $Z_{0}=\mathrm{e}(0)=\mathrm{e}(L(0))$. From the ergodicity of $\tau$ on $\mathrm{T}^{d}$ we may state that for every $\varepsilon>0$ there exist $K \in \mathbb{Z}$ such that $\left\|\tau^{K}(L(0))-L(0)\right\|<\varepsilon$. It follows that for every $\epsilon>0$ there exist some $n \in \mathbb{Z}$ such that $\left\|\mathrm{e} \circ \tau^{n}(0)-\mathrm{e}(0)\right\|<\epsilon$ implying

$$
\forall \epsilon>0, \exists n \in \mathbb{Z}, \quad\left\|Z_{n T}-Z_{0}\right\|<\epsilon,
$$

i.e., $Z_{k T}$ is quasi-periodic. One concludes that the initial shape of the approximated state will up to some small error reoccur at some multiple of the classical period if the Floquet exponents of the flow differential are purely imaginary. We have again no à priori reasons to exclude unbounded motion.

In the case of a periodic flow, we have localization at the initial point and the initial profile (up to some small error) at classical periods. For bounded classical motion, those recurrences define revivals at periods of the classical flow that have already been described [11] in the past.

We summarize our findings in the following theorem.

Theorem 2. Under the following assumptions

(1) $H(X)$ satisfies the conditions of section 1.1

(2) $H^{\prime \prime}\left(X_{t}\right)$ is T-periodic and $\nu$ is the maximal real part of the Floquet exponents of the flow differential, solution of eq. (8),

and with $\kappa:=2 T \sup _{t \in[0, T]}\left\|\mathcal{J} H^{\prime \prime}\left(X_{t}\right)\right\|_{\mathrm{HS}}$ where $K:=e^{\kappa}$, we can make the following statements up to $\mathcal{O}\left(\hbar^{\alpha}\right), \alpha>0$ as $\hbar \searrow 0$.

If $K$ is small enough then the approximation described by theorem 11 will hold up to times $\frac{|\ln (\hbar)|}{6 \nu}$ and the approximate width of the state will behave like $e^{2 \nu|t|}$. 
In particular, if the Floquet exponents are purely complex or zero then the semiclassical propagation described in theorem 1 will hold to times $\hbar^{-\frac{1}{2}}$. The width of the approximated state will be bounded and recurrences will, up to small errors, take place at a multiple of $T$.

Aknowledgement 1. We would particularly like to thank Jens Bolte for valuable comments and discussions.

\section{REFEREnCES}

[1] M. Combescure and D. Robert. Semiclassical spreading of quantum wave packets and applications near unstable fixed points of the classical flow. Asymptot. Anal., 14(4):377-404, 1997.

[2] Thierry Paul and Alejandro Uribe. A construction of quasi-modes using coherent states. Ann. Inst. H. Poincaré Phys. Théor., 59(4):357-381, 1993.

[3] T. Hartmann, F. Keck, H. J. Korsch, and S. Mossmann. Dynamics of bloch oscillations. New J. Phys., 6(2), 2004.

[4] D. Witthaut, F. Keck, H. J. Korsch, and S. Mossmann. Bloch oscillations in two-dimensional lattices. New J. Phys., 6(41), 2004.

[5] F. Bloch. Über die quantenmechanik der elektronen in kristallgitter. Zeitschrift für Physik.

[6] George A. Hagedorn. Semiclassical quantum mechanics. III. The large order asymptotics and more general states. Ann. Physics, 135(1):58-70, 1981.

[7] George A. Hagedorn. Semiclassical quantum mechanics. IV. Large order asymptotics and more general states in more than one dimension. Ann. Inst. H. Poincaré Phys. Théor., 42(4):363-374, 1985.

[8] Gerald B. Folland. Harmonic analysis in phase space, volume 122 of Annals of Mathematics Studies. Princeton University Press, Princeton, NJ, 1989.

[9] G. Floquet. Sur les équations différentielles linéaires à coefficients périodiques. Ann. Sci. École Norm. Sup. (2), 12:47-88, 1883.

[10] Earl A. Coddington and Norman Levinson. Theory of ordinary differential equations. McGraw-Hill Book Company, Inc., New York-Toronto-London, 1955.

[11] I.Sh. Averbuck and N.F. Perelman. Fractional revivals: universality in the long-term evolution of quantum wave packets beyond the correspondence principle dynamics. Physics Letters A, 139:449-453, 1989.

Institut für Theoretische Physik, Universität Ulm, Albert-Einstein-Allee 11, D89069-Ulm, Germany

E-mail address: eric.straeng@uni-ulm.de 